\title{
To Say or not to Say: Dyadic Ambivalence over Emotional Expression and its Associations with Pain, Sexuality and Distress in Couples Coping with Provoked Vestibulodynia.
}

Awada, N., Bergeron, S., Steben, M., Hainault, V.A., \& McDuff, P.

\begin{abstract}
Introduction. Provoked vestibulodynia (PVD) is a highly prevalent and taxing female genital pain condition. Despite the intimate nature of this pain and the fact that affective factors such as anxiety have been shown to modulate its manifestations, no study has yet explored the emotional regulation of couples in which the woman suffers from PVD.

Aim. Ambivalence over emotional expression (AEE) is an emotional regulation variable that quantifies the extent to which a person is comfortable with the way s/he expresses emotions. We examined whether the dyadic AEE of couples in which the woman suffers from PVD was differentially associated with women's pain and couples' psychological, sexual and relational functioning.
\end{abstract}

Methods. Couples $(\mathrm{N}=254)$ in which the woman suffered from PVD completed the ambivalence over emotional expression questionnaire. A couple typology of dyadic AEE was created.

Main Outcome Measures. Dependent measures for both members of the couple were the global measure of sexual satisfaction scale, the Beck depression inventory II, and the revised dyadic adjustment scale. The female sexual function index and the sexual history form were used to assess the sexual function of women and men, respectively. Women also completed the pain rating index of the McGill pain questionnaire. 
Results. Couples in which both partners were considered low on AEE had the highest scores on sexual satisfaction $(\mathrm{p}=.02)$ and function $(\mathrm{p}<.01)$, the lowest depression scores $(\mathrm{p}<.01)$, and the best dyadic adjustment $(\mathrm{p}=.02)$. No difference in pain intensity was found between couples. Conclusions. Findings suggest that, for couples in which the woman suffers from PVD, an emotional regulation that is low in ambivalence in both partners is associated with better psychological, sexual and relational outcomes. Results indicate that emotional regulation may be important to consider in the assessment and treatment of couples coping with PVD.

\section{Introduction}

Provoked vestibulodynia (PVD) is the most common cause of vulvodynia [1], affecting up to $12 \%$ of pre-menopausal women in the community and $15 \%$ of fertile women in gynaecological clinics . Defined by the International Society for the Study of Vulvovaginal Disease (ISSVD) as "vulvar discomfort, occurring in the absence of relevant visible findings or a specific, clinically identifiable, neurologic disorder" [4], PVD pain is localized in the vestibule and triggered by physical contact that can be sexual in nature, such as during intercourse, or not, such as during tampon insertion. Importantly, PVD has been associated with a number of negative sexual consequences, such as decreased sexual function, intercourse frequency, sexual satisfaction, sexual self-efficacy, and sexual self-esteem . Women with PVD also experience psychological difficulties, reporting more depression and anxiety, as well as a reduction in selfesteem and quality of life when compared to women with no genital pain . In spite of these consequences, important gaps concerning the psychosocial aspects of this condition remain. Although relationship factors such as partner responses appear to play a role in the experience of pain and associated psychosexual sequelae [11], no research to date has explored the emotional regulation of couples coping with PVD. 
A number of studies have examined how biomedical factors may relate to PVD.

Recurrent yeast infections, and sub-optimal pelvic floor muscle function, for instance, have been associated with PVD. Other studies focusing on the role of psychological factors in the experience of pain and disability reported by these women have mostly focused on cognitive variables. Consistent with the larger chronic pain literature, global and stable attributional styles (i.e. thinking of the pain as enduring and affecting one's entire life) are associated with worse sexual, psychological and relational outcomes for women with PVD [15]. Fear avoidance factors such as catastrophizing, fear of pain, and hypervigilance also explain part of the variance in pain and sexual functioning of these women [16]. However, research exploring the emotional regulation of women coping with PVD remains particularly scarce.

Furthermore, the pain associated with PVD often occurring in a sexually intimate context, it becomes important to consider that the partners of these women are the usual witnesses and 'perpetrators' of the pain. Contrary to the afflicted women, their male partners do not seem to show increased levels of sexual dysfunction, psychological distress or dyadic difficulties as compared to norms [17]. However, research which has mostly explored cognitive and behavioral variables points to the importance of attending to both members of couples in which the woman suffers from PVD. Global and stable attributional styles are associated with less sexual satisfaction and dyadic adjustment for the partners ${ }^{[18]}$, much like what was found for their female counterparts. Recently, it was found that partners' facilitative responses (i.e. reactions that encourage women's coping efforts with pain) are associated with less pain and more sexual satisfaction for women with PVD [11]. However, research involving the partners has largely ignored emotional factors that are likely involved in the pain, distress, and disability experienced by these couples. 
This neglect of emotional regulation factors in the study of PVD, a condition which lies at the intersection of chronic pain and impaired sexuality, is quite surprising considering that elements of affective functioning are thought to be central predisposing, maintaining, and/or consequential factors in both domains . One of the only affective factors that has been well studied in relation to vulvodynia is anxiety, shown to be both an antecedent and a consequence of sexual pain [24], and thereby pointing to the relevance of examining emotional regulation in the PVD population.

Ambivalence over Emotional Expression (AEE) is an emotional regulation variable which assumes an interpersonal context of emotional expression, and which is therefore especially suited to examine in couples. It is defined as the extent to which a person is comfortable with the way he or she expresses emotions, independently of the level of expressiveness per se [25]. It thus goes one step beyond merely describing a person as expressive or inexpressive, by gauging what hides behind the style of expression. Is the inexpressive person making an effort to actively inhibit the expression of his or her emotions? Does the expressive person often express emotions that he or she wanted to keep private in the first place? Generally, a person would be qualified as ambivalent over emotional expression when the way in which he or she expresses emotions (or does not) is personally problematic and carries with it negative personal consequences such as feeling inadequate or fearing to hurt someone else.

AEE, as measured by the ambivalence over emotional expression questionnaire (AEQ), has been shown to predict more pain, disability, and psychological distress for patients with chronically painful conditions such as chronic low back pain or gastrointestinal cancer [26, 27]. Studies in which the patients and their partners are included show that AEE predicts their respective anxiety and decrease in life satisfaction [28], pointing again to the relevance of also 
attending to the partners of chronic pain sufferers. In fact, for patients diagnosed with gastrointestinal cancer, it was found that the caretaker's AEE was predictive of an increase in the patient's intensity of pain and pain behaviours and of a decrease in the patient's well-being, independently of the patient's own level of ambivalence [27].

Recently, Ben-Ari \& Lavee [29] suggested that AEE is, in fact, better conceptualized as a dyadic variable, rather than as an individual difference measure. These researchers have found that an individual's AEE could predict his marital quality better than other measures commonly thought to be strongly associated with various interpersonal variables (e.g. neuroticism). More importantly, it was shown that dyadic AEE, or looking at the ambivalence across the couple as a single variable rather than in each individual, could predict relationship quality better than each individual's level of AEE. Overall, couples from the community in which both partners are high in AEE show the worst relationship quality outcomes as compared to couples in which one or both partners are low on AEE. Similar results were found in the chronic pain population: couples in which both partners are highly ambivalent over emotional expression have the worst outcomes in terms of pain, impairment, and psychological distress [27, 28].

\section{Aims}

The present study aimed to compare couples based on their dyadic AEE and examine whether they differ on the intensity of pain reported by the women, and levels of sexual satisfaction, sexual functioning, depressive symptoms, and dyadic adjustment reported by the couple as a whole. We expected that couples in which both partners scored highly on AEE would report worse sexual satisfaction, sexual functioning, depression scores, and dyadic adjustment than couples in which both partners were considered low on AEE. We also expected that women 
who were considered to be in high ambivalence couples would report more pain than women who were considered to be in low ambivalence couples. We did not have specific hypotheses concerning couples in which only one partner was considered ambivalent and gender differences within couple types.

\section{Methods}

\section{Participants}

Couples were recruited through the clinics of two gynaecologists from a large metropolitan university hospital, and through references from other health care professionals (53\% of the sample). Announcements were also posted in local newspapers and several websites (40\% of the sample), and some couples were recruited because they had participated in past research projects that had taken place in the same laboratory ( $6 \%$ of the sample). A remaining $1 \%$ of the sample was recruited through word of mouth. About half of the women in our sample, a total of 117, had received a formal diagnosis of PVD from the gynaecologists involved in the study. However, all women were screened using a telephone semi-structured interview in order to ensure that their symptoms were PVD-like. For women, the inclusion criteria were: (1) pain during intercourse lasting for at least 6 months, occurring at a minimum of $75 \%$ of intercourse attempts, and a source of subjective distress, (2) pain limited to intercourse and other activities in which pressure is exerted on the entry of the vagina (i.e. vulvar vestibule), (3) pain localized and limited to the vulvo-vaginal area, and finally (4) being in a committed relationship for a minimum duration of six months. Exclusion criteria were: (1) vulvar pain not limited to penetration or to an exerted pressure on the vulvo-vaginal area and (2) the presence of any of the following conditions: serious medical or psychiatric disorder, active infection, vaginismus, vulvo-vaginal dermatological lesions, pregnancy, or being younger than 18 years old. Men were 
recruited by asking their female partners whether they would be interested in participating, the only exclusion criteria being an age below 18 years and/or having a serious medical or psychiatric disorder. Of the 274 couples who were eligible and participated in the study, 20 had missing data for a complete questionnaire or for more than $10 \%$ of a measure. The final sample size consisted of 254 couples. The only significant difference between couples in which the woman was formally diagnosed with PVD and those in which the woman was screened via a semi-structured interview was that women were younger in the former group $(p=.003)$.

\section{Measures}

Ambivalence over emotional expression (AEE): Men and women's AEE was measured with the ambivalence over emotional expression questionnaire (AEQ) [25]. This self-report measure consists of 28 items, with the total score being computed on a five-point scale and higher scores indicating more AEE. The AEQ has been shown to have good psychometric properties, including good internal stability $(\alpha=.89)$, test-retest reliability and convergent validity [25]. While this questionnaire has not yet been validated in French, it had a very high internal consistency in our sample $(\alpha=.93)$ and a similar factorial structure than that of the original questionnaire.

\section{Main outcome measures}

Pain: Women's pain was assessed with the pain rating index (PRI) of the McGill pain questionnaire with reference to the vulvo-vaginal pain during intercourse in the last six months [30]. This multidimensional scale is a widely used measure consisting of an adjective list that women rate as qualifying their pain or not. Higher scores indicate more severe pain. This measure has been shown to have very good psychometric properties, including good test-retest reliability, discriminant validity and sensitivity to treatment [31]. The French version of this 
questionnaire has previously been validated [32] and the internal consistency for our sample was $\operatorname{high}(\alpha=.79)$.

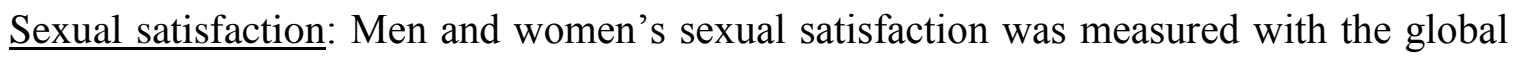
measure of sexual satisfaction scale (GMSEX) [33]. This scale consists of five items yielding a total score from 5 to 35 with higher scores indicating greater satisfaction. This measure has been shown to have good psychometric properties, including good internal consistency $(\alpha=.90)$, testretest reliability and convergent validity [33]. The French version of the test has previously been used with French-speaking participants with an excellent internal consistency $(\alpha=.92)[15]$, a finding which was replicated in our sample $(\alpha=.90)$.

Sexual functioning: Women's sexual functioning was measured with the female sexual function index (FSFI). This questionnaire consists of 19 items measuring five components of sexuality: desire, lubrication, orgasm, satisfaction, and pain - and is most suitable for women who have engaged in sexual activity in the past four weeks $(N=224)$ [34]. Total scores range from 2 to 36 with higher scores indicating better functioning. This questionnaire has been shown to have good psychometric properties, including good internal consistency $(\alpha>.82)$, test-retest reliability, divergent and discriminant validity, as well as being validated with women coping with vulvodynia. The French version of the test has previously been used with a Frenchspeaking population yielding a similar factorial structure as the original version and an excellent internal consistency $(\alpha=.92)$ [15]. The internal consistency for our sample was also high $(\alpha=$ .83). In order to separate sexual impairment from pain, we excluded the pain subscale of the questionnaire in all the analyses.

Men's sexual functioning was measured with the global sexual functioning score of the sexual history form (SHF). This score is calculated using only 12 items of the entire test, chosen 
so as to evaluate different facets of male sexual functioning: frequency of sexual activities, desire, arousal, as well as orgasmic and erectile abilities. It ranges from 0 to 1 , with higher scores indicating worse functioning and has been shown to have good psychometric properties, including excellent test-retest reliability, good internal consistency $(\alpha=.65)$, as well as good discriminant and convergent validity. The French version of the test used in this study has previously been validated [40]. The internal consistency for our sample being below what is typically considered acceptable $(\alpha=.61)$, findings should be interpreted with caution. For the sexual functioning scores of men to be on the same scale and range as the sexual functioning scores of women, we recoded this score into 'newSHF $=((1-\mathrm{SHF}) * 34)+2$ ', and it is this score which is reported in the present paper, with higher scores indicating better functioning.

Depression: Men and women's depressive symptoms were measured with the Beck depression inventory II (BDI-II). This questionnaire consists of 21 items, with total scores ranging from 0 to 63 , and higher scores indicating more depressive symptoms. This measure has been shown to have excellent psychometric properties, including excellent internal consistency $(\alpha=.93)$ and discriminant validity [41]. This test has also been validated with a French speaking population [42], and had a high internal consistency in our sample $(\alpha=.86)$.

Dyadic adjustment: Men's and women's dyadic adjustment were measured with the revised dyadic adjustment scale (R-DAS). This questionnaire consists of 14 items applicable to cohabiting and/or married couples $(\mathrm{N}=207)$, with scores ranging from 0 to 69 and higher scores indicating better dyadic adjustment. This questionnaire has been shown to have good psychometric properties, including good internal stability $(\alpha=.90)$, as well as good discriminant and convergent validity [43]. Also, the French version of the test used in this study has previously been validated [44] and the internal consistency for our sample was high $(\alpha=.83)$. 


\section{Procedure}

Upon being recruited, women and their partners each received questionnaire packages to be returned by mail. These included consent forms, a sociodemographic questionnaire, and the above-mentioned measures of vulvo-vaginal pain (for women, only), ambivalence over emotional expression, sexual satisfaction, sexual function, depression and dyadic adjustment. Follow-up phone calls were conducted every two weeks by a research assistant in order to ensure that the couple was still interested in participating and to answer questions that they might have, to a maximum of five calls. As compensation, participating couples were offered a thirty minute telephone consultation with a sexologist who is part of the research team. This consultation consisted in explaining the diagnosis of PVD: its causes, consequences and the available treatments. The sexologist also answered the couple's questions and referred them to appropriate health care professionals, in addition to sending them educational documentation by email. These procedures were approved by the Institutional Review Boards of the university and university hospital where the research took place.

\section{Couple typology}

As per Porter et al., 2005 [27], median breaks were applied to the AEE scores of men and women, coding ' $\mathrm{H}$ ' for high AEE and ' $\mathrm{L}$ ' for low AEE. Couples were then regrouped into a four-unit typology: $27.6 \%$ were LL couples in which both partners were coded low on AEE; 22.8\% were LH couples in which the woman had ' $\mathrm{L}$ ' AEE and the partner had ' $\mathrm{H}$ ' AEE; $24 \%$ were HL couples in which the woman had ' $\mathrm{H}$ ' AEE and the partner had 'L' AEE; and 25.6\% were $\mathrm{HH}$ couples in with both partners had 'H' AEE.

\section{Data Analysis}


For the couple typology comparison on women's pain intensity, an ANOVA was conducted. For the couple typology comparisons on sexual satisfaction, sexual function, depression scores, and dyadic adjustment, repeated measures ANOVAs were used in order to account for the interdependency of the couples data and pair scores of both members of the same couple. Least significant difference tests were used for post-hoc comparisons. Sociodemographic variables with a correlation superior to .3 with a dependent variable were controlled for in this study [45]. Because the couple was considered the unit of analysis, gender differences were only reported if they were present in the same couple type. Gender differences between couple types were considered beyond the scope of this paper, however, and were not explored.

\section{Results:}

Sample characteristics

Table 1 summarizes the descriptive statistics for this sample. The women had had vulvovaginal pain for an average of more than five years, accurately reflecting the chronicity of this type of pain. They also had significantly higher scores on AEE than their male counterparts ( $t$ $(253)=3.995, \mathrm{p}<.01)$, a result which is consistent with previous research [25]. Finally, the women in our sample were significantly less sexually satisfied $(\mathrm{t}(253)=-2.646, \mathrm{p}=.009)$, sexually functional $(\mathrm{t}(223)=-19.65, \mathrm{p}<.001)$, and more depressed $(\mathrm{t}(253)=8.626, \mathrm{p}<.001)$ than their partners.

\section{Zero-order correlations}

Only a worse sexual function for men was highly correlated with being older $(r=-.36, p$ $<.01)$ and having an older partner $(\mathrm{r}=-.31, \mathrm{p}<.01)$. Because of the very high correlation between ages of men and women in this sample $(r=.88, \mathrm{p}<.01)$, it was decided that only the ages of partners would be controlled for in analyses including their sexual functioning. 
Table 2 presents the intercorrelations between the independent and dependent variables of the study. In accord with our hypotheses, AEE of women was associated with their reduced sexual satisfaction $(\mathrm{r}=-.21, \mathrm{p}<.01)$, sexual function $(\mathrm{r}=-.26, \mathrm{p}<.01)$ and dyadic adjustment $(\mathrm{r}$ $=-.29, \mathrm{p}<.01)$, and with more pain $(\mathrm{r}=.20, \mathrm{p}<.01)$, and higher depression scores for both the women $(\mathrm{r}=.52, \mathrm{p}<.01)$ and the partners $(\mathrm{r}=.14, \mathrm{p}<.05)$. AEE of men was associated with their reduced sexual satisfaction $(-.14, \mathrm{p}<.01)$ and sexual function $(\mathrm{r}=-.16, \mathrm{p}<.01)$ and increased depression scores $(\mathrm{r}=.47, \mathrm{p}<.01)$. Also, it was correlated with a reduced dyadic adjustment for both men $(\mathrm{r}=-.15, \mathrm{p}<.01)$ and women $(\mathrm{r}=-.25, \mathrm{p}<.01)$.

Sexual satisfaction and sexual function of women were highly correlated $(r=.54, \mathrm{p}<$ $.01)$, as were sexual satisfaction of men and women $(\mathrm{r}=.41, \mathrm{p}<.01)$, and sexual satisfaction of men with sexual function of women $(\mathrm{r}=.35, \mathrm{p}<.01)$. Adding the conceptual interdependency to the empirical association of these measures, it was decided that sexual function and satisfaction of men and women would be combined in a same MANOVA in subsequent analyses.

Associations of couple typology with sexual satisfaction, sexual function, depression, dyadic adjustment, and pain

Figures 1 and 2 show the results for sexual satisfaction and sexual functioning scores. A multivariate analysis of covariance (MANCOVA) with repeated measures and controlling for the ages of partners was conducted in order to compare the four types of couples on their sexual satisfaction and sexual function. Main effects of couple type $(F(6,438)=4.150, p=<.001)$, age $(\mathrm{F}(2,218)=7.09, \mathrm{p}<.001)$, and gender $(\mathrm{F}(2,218)=23.865, \mathrm{p}<.001)$ were significant. Further, it was found that the couple typology yielded significant effects for both the sexual satisfaction $(\mathrm{F}(3,219)=3.459, \mathrm{p}=.017)$ and the sexual function of couples $(\mathrm{F}(3,219)=7.139$ 
$\mathrm{p}<.001)$. The simple effect of gender, however, was only significant for sexual function, with women being significantly more sexually impaired than their partners in the four types of couples $(\mathrm{F}(1,219)=41.903, \mathrm{p}<.001)$. Post-hoc analyses showed that 'LL' couples had significantly higher sexual satisfaction and sexual function than the other three types of couples, the latter being statistically equivalent to one another.

Figure 3 shows the results for depression scores. An ANOVA with repeated measures conducted in order to compare the four types of couples on depression scores yielded a significant main effect of gender $(\mathrm{F}(1,250)=80.3$, p <.001), and couple type $(\mathrm{F}(3,250)=15.8$, $\mathrm{p}<.001)$. Notably, there was a significant interaction effect between gender and couple type $(\mathrm{F}$ $(3,250)=11.6, p<.001):$ Women were more depressed than their male partners in 'LL', 'HL' and 'HH' couples, but not in 'LH' couples. The post-hoc analyses showed that 'LL' couples were significantly less depressed than the other three types of couples, and that 'LH' couples were significantly less depressed than 'HH' couples.

Figure 4 shows the results for dyadic adjustment scores. An ANOVA with repeated measures was conducted in order to compare the four types of couples on dyadic adjustment. The main effect of couple type was significant $(F(3,203)=3.54, p=.016)$. Furthermore, there was an interaction effect between gender and couple type $(F(3,203)=3.0, p=.032)$ : Women in 'HH' couples had a significantly lower dyadic adjustment than their partners, a result which was not replicated in any of the other three types of couples. Post-hoc analyses showed that 'HL' and 'HH' couples had significantly reduced dyadic adjustment compared to 'LL' couples.

A univariate analysis of variance (ANOVA) conducted in order to compare the four types of couples on pain intensity was not significant $(F(3,250)=1.301, p=.275)$. 


\section{Discussion:}

Although an increasing number of PVD research has focused on psychosexual variables, studies pertaining to the emotional regulation and dyadic aspects of this sexual pain problem remain scarce. The purpose of this study was to examine the dyadic AEE of couples in which the woman suffers from PVD, and its associations with their sexual satisfaction, sexual function, psychological distress, dyadic adjustment, and pain. In accord with our main hypothesis, we found that couples in which both partners were lower on AEE (LL) were more sexually satisfied and functional, had reduced depression scores, and better relationship adjustment than couples in which both partners were more ambivalent over the expression of their emotions (HH). Women's pain intensity did not differ significantly between the four groups of couples, although women's lower AEE was associated with their reduced pain.

Couples in which both partners were considered low on AEE (LL) were more sexually satisfied than the other three couple types (LH, HL, and HH). High AEE being characterized by a style of emotional expression that is generally accompanied by negative personal consequences and inner conflict, it is likely that the highly ambivalent men and women of our sample experienced this same discomfort when communicating about their sexuality. In fact, general communication apprehension, a closely related variable which concerns the anxieties and fears that may accompany interpersonal communication, has been linked to a reduced satisfaction with sexual communication in a non-clinical sample [46]. Further, better communication about sexuality is a robust correlate of increased sexual satisfaction in both community and clinical samples of men and women. The vulvo-vaginal pain experienced by women in our study and its consequences on the relationship may be an emotionally charged subject that both partners have to communicate about. Couples in which partners are relatively free of AEE (LL) may find it 
easier to manage the sexual pain condition, if only in terms of expressing their sex-related emotions, and negotiating their sexual repertoire and preferences in a less internally conflicted way. This could allow these couples to experience better sexual satisfaction, as compared to couples in which one or both partners experience more AEE. To this effect, sexual intimacy, which broadly qualifies the interaction between members of a couple around sex-related disclosures [51], was positively associated with women's sexual satisfaction in a recent study on PVD. It thus appears that regulating one's emotions may protect against sexual dissatisfaction in couples confronted with PVD.

Women were found to report significantly more sexual dysfunction than their partners in the four types of couples, a result which is not surprising considering their pain during intercourse and the sexual impairment associated with it [6]. Similarly to the results for sexual satisfaction, LL couples were significantly more sexually functional than the other three types of couples (LH, HL, and HH). In a sample of women coping with chronic pelvic pain, it was found that those who were higher on AEE and/or catastrophizing benefited the most from an expressive writing task in terms of their sexual impairment [52]. It is possible that women with PVD who are highly ambivalent are less able to appropriately regulate or communicate their preoccupations and emotions during sex, thereby interfering with their sexual experience and function. Preoccupying thoughts during sex, which have been found to often concern the emotional consequences of engaging in the sexual activity [53], may be particularly charged for men and women dealing with PVD. It is possible that when one or both partners of these couples have relatively high AEE, they will feel more conflicted over expressing these emotional preoccupations and, in turn, also more anxious and less able to refocus on the sexual activity at hand - thereby negatively impacting the sexual function of the couple as a whole [54]. This is in 
accord with the cognitive distraction model of sexual dysfunction [55], which has been found to be relevant to women who suffer from painful intercourse [56]. In an eye-tracking visual attention study, women with dyspareunia were found to spend less time focusing on erotic aspects of images than a control group of women. Interestingly however, women with painful intercourse seemed to not only be distracted away from erotic stimuli, but also actively avoidant of them, likely because of how these relate to their pain-related fear and anxiety [56, 57]. One mechanism that could explain why higher AEE couples coping with PVD have worse sexual function may be that they have more difficulty regulating their preoccupations, fears, and anxiety together during sex, making it harder for them to refocus on erotic, arousing thoughts [58].

LL couples also reported lower depression scores when compared to the other three types of couples. Higher AEE couples could be more psychologically distressed because of their attributions about PVD, whereby in the absence of clearly identifiable physical pathology and treatment, they could be more inclined to blame themselves for the genital pain and/or to see it as enduring and affecting their entire life. Such negative attributions in women with PVD and their partners have been found to predict an increase in their respective psychological distress . It is possible that if more conflicted over their emotional regulation and expression, the negative pain attributions of higher AEE couples could be maintained, leading them to experience guilt, helplessness, and other such negative emotions, and thereby also increasing their depressive symptomatology. Higher levels of AEE and depressive symptoms have also been found to be correlated in student and chronic pain samples, and this relation was partly mediated by catastrophizing in both populations. This may be another pathway that links AEE and psychological distress in couples coping with painful intercourse: catastrophizing is associated with negative overall outcomes for both partners of PVD couples . Higher AEE couples, through 
their emotional regulation difficulties, could come to develop a more catastrophic cognitive appraisal of the genital pain, thereby increasing their distress. Interestingly, in a disease-related chronic pain sample, patient catastrophizing has been found to partially mediate the relationship between the caregiving partner's AEE and the patient's distress[27]. It is perhaps not surprising then that in our study, couples in which only the male partners were considered ambivalent (LH) were less distressed than those in which both partners were $(\mathrm{HH})$. This may indicate that women in our sample who scored lower on AEE contribute, perhaps through a mechanism of reduced patient pain catastrophizing, to diminish the overall emotional distress in the relationship.

Lastly, dyadic adjustment was found to be superior in LL couples when compared to couples in which only the woman or both partners were ambivalent over the expression of their emotions (HL and $\mathrm{HH}$ ). Inexpressive ambivalents in particular have been found to more often interpret facial expressions of emotion with the opposite valence of that which is conveyed [64], which may partly account for the increased relational distress in ambivalent couples. They may be providing and receiving the wrong kind of support to and from their significant others [65]. Importantly, emotional support from one's partner is thought to be a need which is particularly central in chronic pain patients [66]. Ambivalent women have also been found to be less congruent regarding their verbal and nonverbal communications [65], a finding which may contribute to the reduced dyadic adjustment which is reported by couples in which only the woman or both partners are ambivalent (HL and $\mathrm{HH}$ ) as compared to low ambivalence couples (LL). Little is known however about the nonverbal communication correlates of ambivalent men. Finally, it is noteworthy that women were significantly more relationally distressed than their partners in $\mathrm{HH}$ couples. This may reflect their difficulty expressing their need for emotional support for their pain from their partners, who are also ambivalent [67]. 
The fact that dyadic AEE was not associated with pain suggests that couples' levels of AEE are perhaps more relevant to their psychological, sexual, and relational well-being rather than with the intensity of the pain reported by the woman, per se. However, higher AEE of women was significantly associated with their higher pain intensity, a result which is consistent with previous findings in the chronic pain literature [26]. It may be that women who are less comfortable with the way they express their emotions regarding sexual pain could develop a more catastrophic cognitive appraisal of it and also become more oriented towards expressing their physical symptoms instead of their associated emotions. Catastrophizing has, in fact, been found to mediate the relationship between AEE and pain in a non-clinical student sample [59], and associations between increased AEE, catastrophizing, and pain have also been found in chronic pain samples $[27,52]$.

Taken together, findings of the present study indicate that when faced with the challenge of PVD, low ambivalence couples are more sexually satisfied and functional, less psychologically distressed, and more relationally adjusted than high ambivalence couples. One important difference between our results and previous findings concerning dyadic AEE is that in our sample, low ambivalence couples were generally superior in their outcomes to the three other types of couples, whereas in other chronic pain populations, high ambivalence couples stand out as inferior to the three other types of couples in terms of outcomes. This may be due to the highly emotional and intimate nature of the pain experienced by the women in our sample. Pain in the context of sexuality may be particularly difficult to regulate and/or to communicate about for both partners, perhaps especially for those who would generally be qualified as ambivalent over the expression of emotions. 
This study is not without limitations. The cross-sectional design cannot account for causal links or directions between variables. All the measures consisted of self-report questionnaires. Also, not all women in our sample had been diagnosed with PVD by a physician and they were all in stable, mostly cohabiting relationships or married, which may not be generalizable to the PVD population as a whole. Despite these limitations, this study adds to the growing body of research which explores the associations between emotional regulation and the adjustment to various chronic pain conditions, and which includes the caregivers/partners in their conceptualization of the experience of pain $[20,22]$. It is also the first study to explore the emotional regulation of couples coping with PVD. Clinically, results suggest that AEE could be targeted in the assessment and treatment of couples with PVD. Examining how couples regulate their emotions related to painful intercourse could represent an additional therapeutic element, which would complement cognitive-behavioral strategies commonly used in the treatment of this sexual health problem.

\section{Conclusion:}

PVD couples in which both partners were low on ambivalence over emotional expression were more sexually satisfied and functional, less psychologically distressed, and more relationally adjusted than couples in which both partners reported higher ambivalence in the expression of their emotions. Future research should focus on better defining these associations and informing them by examining potential mediators and moderators, such as satisfaction with sexual communication, catastrophizing, and intimacy. 


\section{References}

1. Harlow BL, Stewart EG. A population-based assessment of chronic unexplained vulvar pain: Have we underestimated the prevalence of vulvodynia? J Am Med Wom Assoc 2003;58:82-88.

2. Goetsch MF. Vulvar vestibulitis: Prevalence and historic features in a general gynecologic practice population. Am J Obstet Gynecol 1991;164:1609-1614.

3. Harlow BL, Wise LA, Stewart EG. Prevalence and predictors of chronic lower genital tract discomfort. Am J Obstet Gynecol 2001;185:545-550.

4. Moyal-Barracco M, Lynch PJ. 2003 ISSVD terminology and classification of vulvodynia: A historical perspective. J Reprod Med 2004;49:772-777.

5. Brauer M, Kuile M, Laan E. Effects of appraisal of sexual stimuli on sexual arousal in women with and without superficial dyspareunia. Arch Sex Behav 2009;38:476-485.

6. Desrochers G, Bergeron S, Landry T, Jodoin M. Do psychosexual factors play a role in the etiology of provoked vestibulodynia? A critical review. J Sex Marital Ther 2008;34:198-226.

7. Gates E, Galask R. Psychological and sexual functioning in women with vulvar vestibulitis. Obstet Gynecol 2001;22:221-228.

8. Smith KB, Pukall CF. A systematic review of relationship adjustment and sexual satisfaction among women with provoked vestibulodynia. J Sex Res 2011;48:166-191.

9. Sutton KS, Pukall CF, Chamberlain S. Pain ratings, sensory thresholds, and psychosocial functioning in women with provoked vestibulodynia. J Sex Marital Ther 2009;35:262-281.

10. Arnold LD, Bachmann GA, Rosen R, Kelly S, Rhoads GG. Vulvodynia: Characteristics and associations with comorbidities and quality of life. Obstet Gynecol 2006;107:617-624.

11. Rosen NO, Bergeron S, Glowacka M, Delisle I, Baxter ML. Harmful or helpful: Perceived solicitous and facilitative partner responses are differentially associated with pain and sexual satisfaction in women with provoked vestibulodynia. J Sex Med 2012;9:2351-2360.

12. Sarma AV, Foxman B, Bayirli B, Haefner H, Sobel JD. Epidemiology of vulvar vestibulitis syndrome: an exploratory case-control study. Sex Transm Infect 1999;75:320-326.

13. Bazin S, Bouchard C, Brisson J, Morin C, Meisels A, Fortier M. Vulvar vestibulitis syndrome: An exploratory case-control study. Obstet Gynecol 1994;83:47-50.

14. Bergeron S, Rosen NO, Morin M. Genital pain in women: Beyond interference with intercourse. Pain 2011;152:1223-1225.

15. Jodoin M, Bergeron S, Khalifé S, Dupuis M-J, Desrochers G, Leclerc B. Attributions about pain as predictors of psychological symptomatology, sexual function, and dyadic adjustment in women with vestibulodynia. Arch Sex Behav 2011;40:87-97.

16. Desrochers G, Bergeron S, Khalifé S, Dupuis M-J, Jodoin M. Fear avoidance and self-efficacy in relation to pain and sexual impairment in women with provoked vestibulodynia. Clin J Pain 2009;25:520527.

17. Van Lankveld JJ, Weijenborg PTM, Ter Kuile MM. Psychologic profiles of and sexual function in women with vulvar vestibulitis and their partners. Obstet Gynecol 1996;88:65-70.

18. Jodoin M, Bergeron S, Khalifé S, Dupuis MJ, Desrochers G, Leclerc B. Male partners of women with provoked vestibulodynia: attributions for pain and their implications for dyadic adjustment, sexual satisfaction, and psychological distress. J Sex Med 2008;5:2862-2870. 
19. Basson R. Biopsychosocial models of women's sexual response: applications to management of 'desire disorders'. Sex Relation Ther 2003;18:107-115.

20. Lumley MA, Cohen JL, Borszcz GS, et al. Pain and emotion: a biopsychosocial review of recent research. J Clin Psychol 2011;67:942-968.

21. Fishbain DA, Cutler R, Rosomoff HL, Rosomoff RS. Chronic pain-associated depression: Antecedent or consequence of chronic pain? A review. Clin J Pain 1997;13:116-137.

22. Agar-Wilson $M$, Jackson T. Are emotion regulation skills related to adjustment among people with chronic pain, independent of pain coping? Eur J Pain 2012;16:105-114.

23. Laurent SM, Simons AD. Sexual dysfunction in depression and anxiety: Conceptualizing sexual dysfunction as part of an internalizing dimension. Clin Psychol Rev 2009;29:573-585.

24. Khandker M, Brady SS, Vitonis AF, MacLehose RF, Stewart EG, Harlow BL. The influence of depression and anxiety on risk of adult onset vulvodynia. Int J Wom Health 2011;20:1445-1451.

25. King LA, Emmons RA. Conflict over emotional expression: Psychological and physical correlates. J

Pers Soc Psychol 1990;58:864-877.

26. Carson JW, Keefe FJ, Lowry KP, Porter LS, Goli V, Fras AM. Conflict about expressing emotions and chronic low back pain: Associations with pain and anger. J Pain 2007;8:405-411.

27. Porter LS, Keefe FJ, Lipkus I, Hurwitz H. Ambivalence over emotional expression in patients with gastrointestinal cancer and their caregivers: Associations with patient pain and quality of life. Pain 2005;117:340-348.

28. Tucker JS, Winkelman DK, Katz JN, Bermas BL. Ambivalence over emotional expression and psychological well-being among rheumatoid arthritis patients and their spouses. J Appl Soc Psychol 1999;29:271-290.

29. Ben-Ari A, Lavee Y. Ambivalence over emotional expressiveness in intimate relationships: A shift from an individual characteristic to dyadic attribute. Am J Orthopsychiatry 2011;81:277-284.

30. Melzack R. The McGill Pain Questionnaire: Major properties and scoring methods. Pain 1975;1:277299.

31. Melzack R, Katz J. The McGill Pain Questionnaire: Appraisal and current status. In: Press TG, ed. Handbook of Pain Assessment. New York; 1992:152-168.

32. Boureau F, Luu M, Doubrere JF, Gay C. Elaboration d'un questionnaire d'auto-evaluation de la douleur par liste de qualificatifs. Therapie 1984;39:119-129.

33. Lawrance K, Byers SE. Interpersonal Exchange Model of Sexual Satisfaction Questionnaire. In: Oaks T, ed. Handbook of sexuality-related measures: Sage; 1998:514-519.

34. Meyer-Bahlburg HF, Dolezal C. The Female Sexual Function Index: A methodological critique and suggestions for improvement. J Sex Marital Ther 2007;33:217-224.

35. Rosen R, Brown C, Heiman J, et al. The female sexual function index (FSFI): A multidimensional selfreport instrument for the assessment of female sexual function. J Sex Marital Ther 2000;26:191-208.

36. Wiegel M, Meston C, Rosen R. The female sexual function index (FSFI): Cross-validation and development of clinical cutoff scores. J Sex Marital Ther 2005;31:1-20.

37. Likes WM, Stegbauer C, Hathaway D, Brown C, Tillmanns T. Use of the female sexual function index in women with vulvar intraepithelial neoplasia. J Sex Marital Ther 2006;32:255-266.

38. Masheb RM, Lozano-Blanco C, Kohorn El, Minkin MJ, Kerns RD. Assessing sexual function and dyspareunia with the Female Sexual Function Index (FSFI) in women with vulvodynia. J Sex Marital Ther 2004;30:315-324.

39. Creti L, Fichten CS, Amsel R, et al. Global sexual functioning: a single Summary score for Nowinski and LoPiccolo's Sexual History Form (SHF). In: Davis CM, Yarber WL, Bauserman R, Schreer G, Davis SL, eds. Handbook of sexuality-related measures. Thousand Oaks: Sage Publications; 1998:261-267.

40. Trudel G, Ravart M, Matte B. The use of multiaxial diagnostic system for sexual dysfunctions in assessment of hypoactive sexual desire. J Sex Marital Ther 1993;19:123-130. 
41. Beck AT, Steer RA, Brown GK. BDI-II Manual. San Antonio: Harcourt Brace \& Company; 1996. 42. Bourque $P$, Beaudette $D$. Étude psychometrique du questionnaire de dépression de Beck auprès d'un échantillon d'étudiants universitaires francophones. Can J Behav Sci 1982;14:211-218.

43. Busby DM, Christensen C, Crane DR, Larson JH. A revision of the Dyadic Adjustment Scale for use with distressed and nondistressed couples: Construct hierarchy and multidimensional scales. J Marital Fam Ther 1995;21:289-308.

44. Sabourin S, Valois P, Lussier Y. Development and validation of a brief version of the dyadic adjustment scale with a nonparametric item analysis model. Psychol Assess 2005;17:15.

45. Frigon J-Y, Laurencelle L. Analysis of covariance: A proposed algorithm. Educ Psychol Meas 1993;53:1-18.

46. Wheeless LR, Parsons LA. What you feel is what you might get: Exploring communication apprehension and sexual communication satisfaction. Comm Res 1995;12:39-45.

47. Hayes RD, Dennerstein L, Bennett CM, Sidat M, Gurrin LC, Fairley CK. Risk factors for female sexual dysfunction in the general population: exploring factors associated with low sexual function and sexual distress. J Sex Med 2008;5:1681-1693.

48. MacNeil S, Byers ES. Role of sexual self-disclosure in the sexual satisfaction of long-term heterosexual couples. J Sex Res 2009;46:3-14.

49. Montesi JL, Conner BT, Gordon EA, Fauber RL, Kim KH, Heimberg RG. On the relationship among social anxiety, intimacy, sexual communication, and sexual satisfaction in young couples. Arch Sex Behav 2013;42:81-91.

50. Timm TM, Keiley MK. The effects of differentiation of self, adult attachment, and sexual communication on sexual and marital satisfaction: A path analysis. J Sex Marital Ther 2011;37:206-223. 51. Bois K, Bergeron S, Rosen NO, McDuff P, Grégoire C. Sexual and relationship intimacy among women with provoked vestibulodynia and their partners: Associations with sexual satisfaction, sexual function, and pain self-efficacy. J Sex Med 2013;10:2024-2035.

52. Norman SA, Lumley MA, Dooley JA, Diamond MP. For whom does it work? Moderators of the effects of written emotional disclosure in a randomized trial among women with chronic pelvic pain. Psychosom Med 2004;66:174-183.

53. Purdon C, Holdaway L. Non-erotic thoughts: Content and relation to sexual functioning and sexual satisfaction. J Sex Res 2006;43:154-162.

54. McCabe M, Althof SE, Assalian P, et al. Psychological and interpersonal dimensions of sexual function and dysfunction. J Sex Med 2010;7:327-336.

55. Barlow DH. Causes of sexual dysfunction: The role of anxiety and cognitive interference. J Consult Clin Psychol 1986;54:140.

56. Lykins $A D$, Meana $M$, Minimi J. Visual attention to erotic images in women reporting pain with intercourse. J Sex Res 2010;48:43-52.

57. Basson R. The recurrent pain and sexual sequelae of provoked vestibulodynia: A perpetuating cycle. J Sex Med 2012;9:2077-2092.

58. Nelson AL, Purdon C. Non-erotic thoughts, attentional focus, and sexual problems in a community sample. Arch Sex Behav 2011;40:395-406.

59. Lu Q, Uysal A, Teo I. Need satisfaction and catastrophizing: Explaining the relationship among emotional ambivalence, pain, and depressive symptoms. J Health Psychol 2011;16:819-827.

60. Desrosiers M, Bergeron S, Meana M, Leclerc B, Binik YM, Khalifé S. Psychosexual characteristics of vestibulodynia couples: Partner solicitousness and hostility are associated with pain. J Sex Med 2008;5:418-427.

61. Lemieux AJ, Bergeron S, Steben M, Lambert B. Do romantic partners' responses to entry dyspareunia affect women's experience of pain? The roles of catastrophizing and self-efficacy. J Sex Med 2013;10:2274-2284. 
62. Rosen NO, Bergeron S, Lambert B, Steben M. Provoked vestibulodynia: Mediators of the associations between partner responses, pain, and sexual satisfaction. Arch Sex Behav 2013;42:129-141. 63. Thomtén J, Linton SJ. A psychological view of sexual pain among women: Applying the fearavoidance model. Int J Wom Health 2013;9:251-263.

64. King LA. Ambivalence over emotional expression and reading emotions in situations and faces. J Pers Soc Psychol 1998;74:753-762.

65. Mongrain M, Vettese LC. Conflict over emotional expression: Implications for interpersonal communication. Pers Soc Psychol Bull 2003;29:545-555.

66. Miller J, Timson D. Exploring the experiences of partners who live with a chronic low back pain sufferer. Health Soc Care Comm 2004;12:34-42.

67. King LA. Emotional expression, ambivalence over expression, and marital satisfaction. J of Soc and Pers Relationships 1993;10:601-607. 